\title{
Signatures of the Higgs mode in transport through a normal-metal-superconductor junction
}

\author{
Gaomin Tang $\odot,{ }^{1}$ Wolfgang Belzig $\odot,{ }^{2}$ Ulrich Zülicke $\odot,{ }^{3}$ and Christoph Bruder ${ }^{1}$ \\ ${ }^{1}$ Department of Physics, University of Basel, Klingelbergstrasse 82, CH-4056 Basel, Switzerland \\ ${ }^{2}$ Fachbereich Physik, Universität Konstanz, D-78457 Konstanz, Germany \\ ${ }^{3}$ School of Chemical and Physical Sciences and MacDiarmid Institute for Advanced Materials and Nanotechnology, \\ Victoria University of Wellington, P.O. Box 600, Wellington 6140, New Zealand
}

(Received 21 February 2020; revised manuscript received 5 June 2020; accepted 8 June 2020; published 29 June 2020)

\begin{abstract}
A superconductor subject to electromagnetic irradiation in the terahertz range can show amplitude oscillations of its order parameter. However, coupling this so-called Higgs mode to the charge current is notoriously difficult. We propose to achieve such a coupling in a particle-hole-asymmetric configuration using a DC-voltagebiased normal-metal-superconductor tunnel junction. Using the quasiclassical Green's function formalism, we demonstrate three characteristic signatures of the Higgs mode: (i) The AC charge current exhibits a pronounced resonant behavior and is maximal when the radiation frequency coincides with the order parameter. (ii) The AC charge current amplitude exhibits a characteristic nonmonotonic behavior with increasing voltage bias. (iii) At resonance for large voltage bias, the AC current vanishes inversely proportional to the bias. These signatures provide an electric detection scheme for the Higgs mode.
\end{abstract}

DOI: 10.1103/PhysRevResearch.2.022068

Introduction. Manipulating the superconducting (SC) state using tailored light pulses is currently receiving a great deal of attention. Various fascinating phenomena have been reported, including superconductivity enhancement [1-6], lightinduced superconductivity [7-10], the presence of chiral Majorana modes in chiral superconductors [11], and the emergence of the Higgs mode [12-38].

The Higgs mode is a gapped collective excitation consisting of the oscillation of the order parameter amplitude in a system with spontaneous symmetry breaking [12-15]. In a superconductor where the $U(1)$ symmetry is spontaneously broken, the order parameter amplitude can oscillate when the system is coupled to external gauge fields. The presence of a Higgs mode in superconductors with charge density waves was first observed using the Raman-scattering technique [16-18] and later theoretically interpreted $[19,20]$. However, the excitation and detection of the Higgs mode in superconductors without charge density waves became experimentally possible only in the last decade due to the experimental advance of ultrafast low-energy terahertz $(\mathrm{THz})$ spectroscopy. Clear nonlinear optical signatures indicating the presence of a Higgs mode have been observed using the pump-probe technique in both $s$-wave $[21-24,26]$ and $d$-wave [27] superconductors. Since the Higgs mode is a scalar excitation, it is expected to couple to the external electromagnetic field in a nonlinear way. A linear coupling enabled by the presence of a supercurrent was theoretically

Published by the American Physical Society under the terms of the Creative Commons Attribution 4.0 International license. Further distribution of this work must maintain attribution to the author $(s)$ and the published article's title, journal citation, and DOI. proposed [30] and experimentally verified [31]. Very recently, it was theoretically predicted that a Higgs mode can be observed through its effect in the time-dependent spin current in a ferromagnet-superconductor junction [28].

In this work, we consider a junction between a normal metal and an s-wave superconductor [see Fig. 1(a)]. THz electromagnetic irradiation can excite a Higgs mode on the SC part of the junction. To couple the Higgs mode to the charge current, particle-hole symmetry has to be broken. Inducing a finite spin splitting in the superconductor is one way to achieve this [28]. As a potentially simpler alternative, we propose to apply a DC voltage bias to a normal-metalsuperconductor (NS) junction. We demonstrate that the Higgs mode will manifest itself in intriguing properties of the AC charge current through the junction [see Fig. 1(c)].

Higgs mode in the superconductor. We consider a superconductor subject to monochromatic electromagnetic irradiation with the time-dependent vector potential $\mathbf{A}(t)=\mathbf{A}_{\Omega} e^{-i \Omega t}$ in the Coulomb gauge. Due to the nonlinear coupling between the electromagnetic field and the Higgs mode, there will be a second-harmonic correction to the static SC gap $\Delta_{0}$. Thus, the time-dependent order parameter can be expressed as

$$
\Delta(t)=\Delta_{0}+\Delta_{2} e^{-i 2 \Omega t} .
$$

If the irradiation frequency is close to $\Delta_{0}$, superconductivity can possibly be enhanced [3,4], and a small correction to the static order parameter can be incorporated in $\Delta_{0}$.

We employ the quasiclassical Green's function technique to study the dynamics of the superconductor and the transport properties of the NS junction. The quasiclassical Green's function $\breve{g}_{s}\left(t, t^{\prime}\right)$ for a dirty superconductor with the diffusion constant $D$ fulfills the time-dependent Usadel equation $[39,40]$

$$
i\left\{\check{\tau}_{3} \partial_{t}, \check{g}_{s}\right\}+\left[\check{\Delta}, \check{g}_{s}\right]-i D \nabla\left(\check{g}_{s} \circ \nabla \check{g}_{s}\right)=0,
$$


(a)
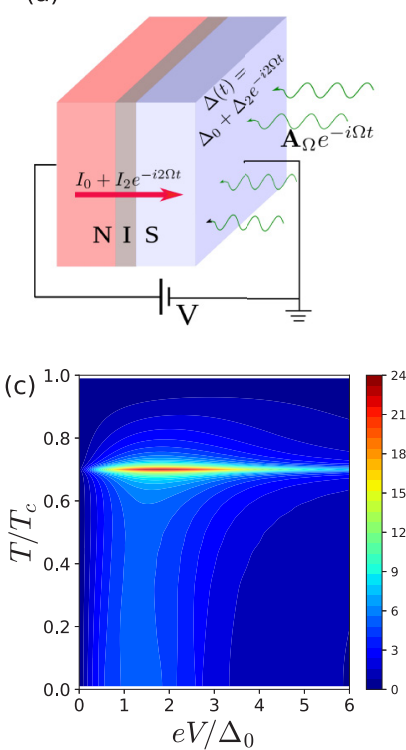
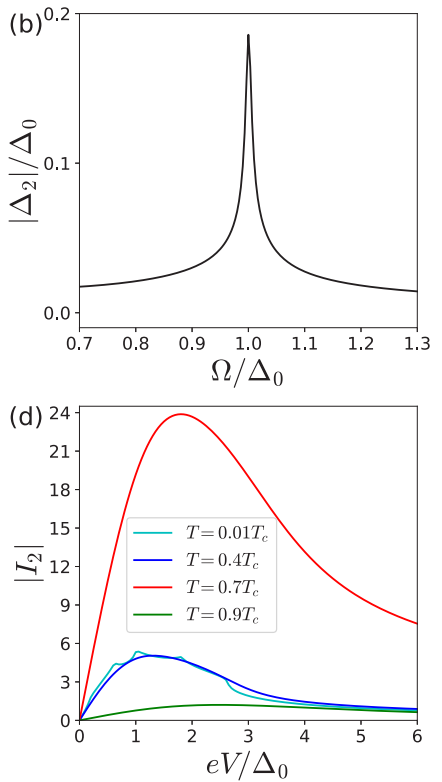

FIG. 1. (a) DC-voltage-biased normal-metal-superconductor (NS) junction with a thin insulator (I) acting as a tunnel barrier. The superconductor is subject to electromagnetic irradiation described by a time-dependent vector potential $\mathbf{A}_{\Omega} e^{-i \Omega t}$, which generates a Higgs mode inside the superconductor. (b) $\left|\Delta_{2}\right| / \Delta_{0}$ as a function of $\Omega / \Delta_{0}$. The parameters are chosen as $T=0.05 T_{c}, \gamma=\left.0.01 \Delta_{0}\right|_{T=0}$, and $W_{A}=\left.0.01 \Delta_{0}\right|_{T=0}$. (c) AC charge current $\left|I_{2}\right|$ plotted against temperature $T$ and bias voltage $\mathrm{eV}$ scaled by the static SC gap amplitude at temperature $T$. The charge currents are in units of $G_{t} W_{A} / e$. The electromagnetic frequency is set to be $\Omega=\left.\Delta_{0}\right|_{T=0.7 T_{c}}$. (d) Line cuts of (c) at different temperatures.

where the Green's function is written in Keldysh space and has the structure

$$
\check{g}_{s}=\left(\begin{array}{cc}
\hat{g}_{s}^{r} & \hat{g}_{s}^{k} \\
0 & \hat{g}_{s}^{a}
\end{array}\right)
$$

The order parameter has the form $\check{\Delta}=i \check{\tau}_{2} \Delta$. The matrices $\check{\tau}_{\alpha}$ with $\alpha=1,2,3$ are diagonal matrices with entries $\hat{\tau}_{\alpha}$, that is, $\check{\tau}_{\alpha}=\operatorname{diag}\left[\hat{\tau}_{\alpha}, \hat{\tau}_{\alpha}\right]$, where $\hat{\tau}_{\alpha}$ are the Pauli matrices in Nambu space. In Eq. (2), the anticommutator is defined as $\left\{\check{\tau}_{3} \partial_{t}, \check{g}_{s}\right\}=$ $\check{\tau}_{3} \partial_{t} \check{g}_{s}\left(t, t^{\prime}\right)+\partial_{t^{\prime}} \check{g}_{s}\left(t, t^{\prime}\right) \check{\tau}_{3}$, and $\nabla \cdot=\partial_{\mathbf{r}} \cdot-i e\left[\check{\tau}_{3} \mathbf{A}(t), \cdot\right]$. The convolution operation between two objects $f$ and $g$ is defined as $(f \circ g)\left(t, t^{\prime}\right)=\int d t_{1} f\left(t, t_{1}\right) g\left(t_{1}, t^{\prime}\right)$. The Green's function obeys the normalization condition $\check{g}_{s} \circ \check{g}_{s}=1$.

Since the Higgs mode couples to the electromagnetic field nonlinearly, to leading order there is a second-harmonic correction $\check{g}_{2}$ to the stationary Green's function $\check{g}_{0}$,

$$
\check{g}_{s}\left(t, t^{\prime}\right)=\check{g}_{0}\left(t-t^{\prime}\right)+\check{g}_{2}\left(t, t^{\prime}\right) \text {. }
$$

The Fourier transforms of $\check{g}_{0}$ and $\check{g}_{2}$ are, respectively, defined as

$$
\begin{aligned}
\check{g}_{0}\left(t-t^{\prime}\right) & =\int \frac{d \epsilon}{2 \pi} e^{-i \epsilon\left(t-t^{\prime}\right)} \check{g}_{0}(\epsilon), \\
\check{g}_{2}\left(t, t^{\prime}\right) & =\int \frac{d \epsilon}{2 \pi} e^{-i \epsilon_{+} t+i \epsilon_{-} t^{\prime}} \breve{g}_{2}\left(\epsilon_{+}, \epsilon_{-}\right),
\end{aligned}
$$

with $\epsilon_{ \pm}=\epsilon \pm \Omega$. The normalization condition leads to $\check{g}_{2}\left(\epsilon_{+}, \epsilon_{-}\right) \check{g}_{0}\left(\epsilon_{-}\right)+\check{g}_{0}\left(\epsilon_{+}\right) \check{g}_{2}\left(\epsilon_{+}, \epsilon_{-}\right)=0$.

For the stationary quasiclassical Green's functions, we have [40]

$$
\begin{gathered}
\hat{g}_{0}^{r(a)}(\epsilon)=g_{0}^{r(a)}(\epsilon) \hat{\tau}_{3}+i \hat{\tau}_{2} f_{0}^{r(a)}(\epsilon), \\
\hat{g}_{0}^{k}(\epsilon)=\left[\hat{g}_{0}^{r}(\epsilon)-\hat{g}_{0}^{a}(\epsilon)\right] \tanh (\beta \epsilon / 2),
\end{gathered}
$$

where $\beta=1 /\left(k_{B} T\right)$, and

$$
g_{0}^{r(a)}=f_{0}^{r(a)} \epsilon / \Delta_{0}=\epsilon / s_{0}^{r(a)},
$$

with $s_{0}^{r(a)}=i \sqrt{\Delta_{0}^{2}-(\epsilon \pm i \gamma)^{2}}$. Here, $\gamma$ is the phenomenological Dynes broadening parameter. Inserting Eqs. (1) and (4) into the Usadel equation (2) leads to the retarded and advanced components of the nonstationary term [28],

$$
\hat{g}_{2}^{r(a)}\left(\epsilon_{+}, \epsilon_{-}\right)=\hat{g}_{V}^{r(a)}\left(\epsilon_{+}, \epsilon_{-}\right)+\hat{g}_{H}^{r(a)}\left(\epsilon_{+}, \epsilon_{-}\right) .
$$

The first term $\hat{g}_{V}$ is due to the direct second-order coupling to the vector potential and reads

$$
\hat{g}_{V}^{r(a)}\left(\epsilon_{+}, \epsilon_{-}\right)=i W_{A}\left[\frac{\hat{\bar{g}}_{0}(\epsilon)-\hat{g}_{0}\left(\epsilon_{+}\right) \hat{\bar{g}}_{0}(\epsilon) \hat{g}_{0}\left(\epsilon_{-}\right)}{s_{+}+s_{-}}\right]^{r(a)},
$$

with $W_{A}=D e^{2}\left|\mathbf{A}_{\Omega}\right|^{2}$ and $\hat{\bar{g}}_{0}=\hat{\tau}_{3} \hat{g}_{0} \hat{\tau}_{3}$. The second term $\hat{g}_{H}$ describes the effect of the Higgs mode,

$$
\hat{g}_{H}^{r(a)}\left(\epsilon_{+}, \epsilon_{-}\right)=i \Delta_{2}\left[\frac{\hat{\tau}_{2}-\hat{g}_{0}\left(\epsilon_{+}\right) \hat{\tau}_{2} \hat{g}_{0}\left(\epsilon_{-}\right)}{s_{+}+s_{-}}\right]^{r(a)} .
$$

Both here and in (10), we have used the notation $s_{+}^{r(a)}=$ $i \sqrt{\Delta_{0}^{2}-\left(\epsilon_{+} \pm i \gamma\right)^{2}}$ and $s_{-}^{r(a)}=i \sqrt{\Delta_{0}^{2}-\left(\epsilon_{-} \pm i \gamma\right)^{2}}$.

An expression for the oscillating part $\Delta_{2}$ of the SC gap can be obtained from the gap equation $\Delta(t)=-i \lambda \operatorname{Tr}\left[\hat{\tau}_{2} \hat{g}_{s}^{k}(t)\right]$, where $\lambda$ is the pairing interaction. The details of the derivation can be found in the Supplemental Material [41]. The static order parameter $\Delta_{0}$ at temperature $T$ (denoted by $\left.\Delta_{0}\right|_{T}$ ) can be well fitted by the interpolation formula

$$
\left.\Delta_{0}\right|_{T}=\left.\Delta_{0}\right|_{T=0} \tanh \left(1.74 \sqrt{T_{c} / T-1}\right),
$$

where $T_{c}$ is the critical temperature. To simplify the notation, we will omit the variable $T$ in $\left.\Delta_{0}\right|_{T}$ at nonzero temperature.

The Dynes broadening parameter and irradiation intensity are fixed as $\gamma=\left.0.01 \Delta_{0}\right|_{T=0}$ and $W_{A}=\left.0.01 \Delta_{0}\right|_{T=0}$ in this work. In Fig. 1(b), we plot the dependence of $\Delta_{2}$ on the irradiation frequency $\Omega$ at temperature $T=0.05 T_{c}$. A cusp appears at $\Omega=\Delta_{0}$ (resonant condition) where the oscillation amplitude of the order parameter $\left|\Delta_{2}\right|$ is maximal. The resonant behavior is a signature of the Higgs mode and can be identified in nonlinear optical response using the pump-probe technique [21-24,26].

Normal-metal-superconductor junction. In the following, we study the transport properties of a DC-voltage-biased NS junction, in which the SC side is subject to electromagnetic irradiation described by a vector potential $\mathbf{A}(t)=\mathbf{A}_{\Omega} e^{-i \Omega t}$ [see Fig. 1(a)]. The THz electromagnetic field is assumed to exist only on the SC side to avoid possible photon-assisted tunneling processes, and its wave vector is parallel to the transport direction of the junction. The thickness of the superconductor is assumed to be smaller than or comparable to the SC coherence length, so that the order parameter can be 
treated as homogeneous. For simplicity, we consider a tunnel junction, which is characterized by its conductance $G_{t}$. The retarded, advanced, and Keldysh components of the Green's function for the normal metal are expressed as

$$
\hat{g}_{n}^{r}=-\hat{g}_{n}^{a}=\hat{\tau}_{3},
$$

and

$$
\hat{g}_{n}^{k}(\epsilon)=2 \operatorname{diag}\left[\tanh \left(\frac{\epsilon-e V}{2 k_{B} T}\right),-\tanh \left(\frac{\epsilon+e V}{2 k_{B} T}\right)\right],
$$

where $V$ is the external voltage bias.

Since the leading perturbation due to the Higgs mode is a second harmonic in $\Omega$, the electric current $I(t)$ can be decomposed in a DC component $I_{0}$ and an AC component $I_{2}$ with $I(t)=I_{0}+I_{2} e^{-i 2 \Omega t}$. For a tunnel junction, the $\mathrm{AC}$ component of the particle current to the first order in the tunnel conductance is

$$
I_{2}=\frac{G_{t}}{8 e} \int d \epsilon \operatorname{Tr}\left(\hat{\tau}_{3} \hat{X}\right)
$$

with

$$
\hat{X}=\hat{g}_{n}^{k}\left(\epsilon_{+}\right) \hat{g}_{2}^{a}\left(\epsilon_{+}, \epsilon_{-}\right)-\hat{g}_{2}^{r}\left(\epsilon_{+}, \epsilon_{-}\right) \hat{g}_{n}^{k}\left(\epsilon_{-}\right) .
$$

Equation (15) implies that $\left.I_{2}\right|_{e V=0}=0$, since the situation is particle-hole symmetric in the absence of a DC voltage bias. A finite voltage bias can break the symmetry so that the Higgs mode can couple to the charge current. This is in contrast to Ref. [28], where particle-hole asymmetry is due to the exchange field in the superconductor induced by an external magnetic field. A detailed derivation of $I_{2}$ using circuit theory [42-44] can be found in the Supplemental Material [41].

Numerical results. The gap oscillation amplitude $\Delta_{2}$ is a complex number. This also applies to the AC charge current $I_{2}$, and we focus on discussing the AC charge current magnitude $\left|I_{2}\right|$. Figures 2(a)-2(c) show the dependence of $\left|I_{2}\right|$ on the voltage bias $\mathrm{eV}$ and the electromagnetic frequency $\Omega$, where panels (b) and (c) are line cuts of panel (a) for different values of $\Omega$ and $e V$, respectively. To illustrate the influence of the Higgs mode on transport, we artificially set $\Delta_{2}=0$ in $\check{g}_{2}$, that is, $\hat{g}_{2}=\hat{g}_{V}$ from Eq. (9), and calculate the corresponding AC current amplitude $\left|I_{2}^{\Delta_{2}=0}\right|$ for comparison [see Figs. 2(d)2(f)]. The charge currents are shown in units of $G_{t} W_{A} / e$.

As can be seen from Fig. 2, the AC charge current amplitude $\left|I_{2}\right|$ at a fixed voltage bias shows a pronounced resonant behavior as a function of frequency at $\Omega=\Delta_{0}$, which is not present for $\left|I_{2}^{\Delta_{2}=0}\right|$. This can be easily observed by comparing Figs. 2(c) and 2(f) at $e V=\Delta_{0}$. Also, $\left|I_{2}\right|$ is much larger than $\left|I_{2}^{\Delta_{2}=0}\right|$ at resonance, since the SC gap oscillation amplitude $\left|\Delta_{2}\right|$ achieves its maximum and is much larger than $W_{A}$ as can be seen from Fig. 1(b). Thus, the Higgs mode dominates the AC charge current at resonance. In Fig. S1 in the Supplemental Material [41], we compare the contributions from $\hat{g}_{V}$ and $\hat{g}_{H}$ in the AC charge current amplitude and find that the contribution from $\hat{g}_{V}$ can be even larger than that from $\hat{g}_{H}$ away from resonance. The resonant behavior of $\left|I_{2}\right|$ can serve as a signature of the presence of the Higgs mode in the superconductor. A similar observation has been recently reported for spin currents driven by the Higgs mode [28].

Figure 2(b) shows that $\left|I_{2}\right|$ exhibits an interesting nonmonotonic behavior: it increases with increasing voltage bias
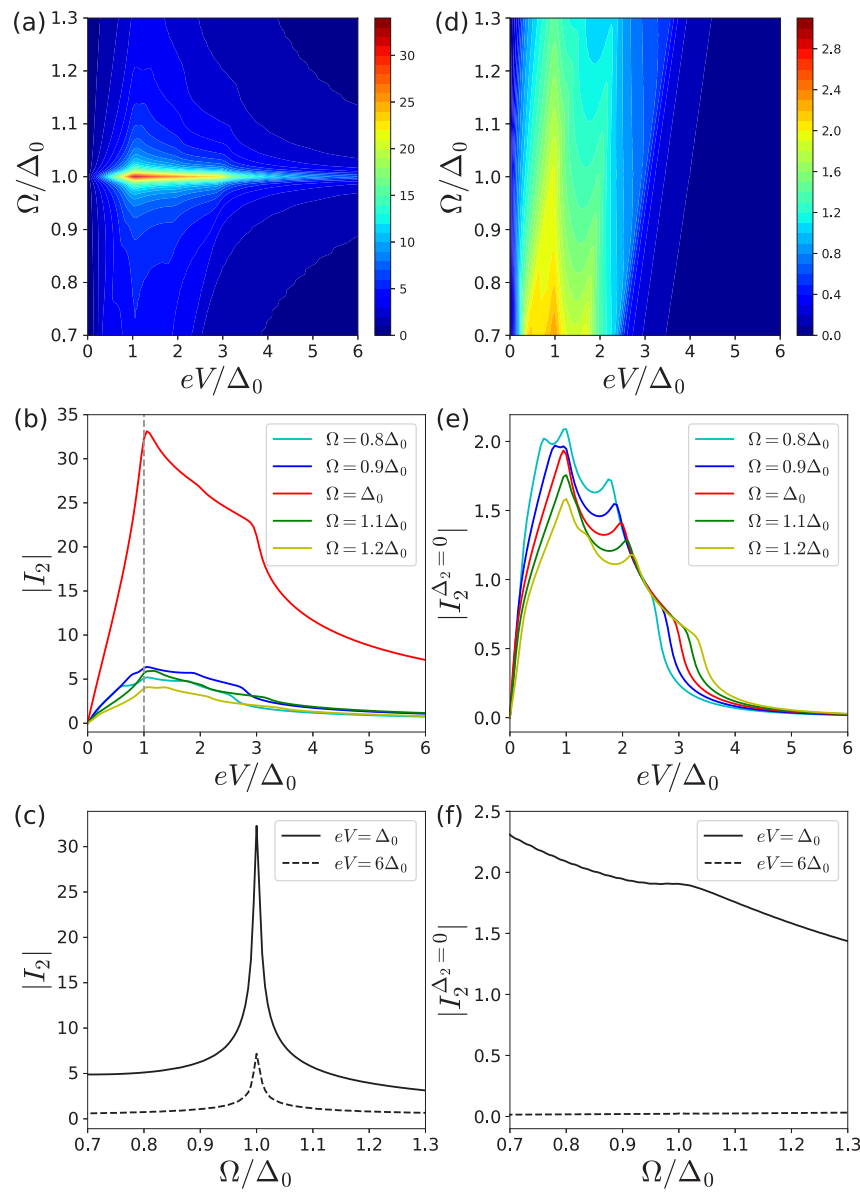

FIG. 2. (a) AC charge current $\left|I_{2}\right|$ plotted against voltage bias $\mathrm{eV}$ and irradiation frequency $\Omega$. Panels (b) and (c) are line cuts of (a) at different $\Omega$ and $e V$, respectively. (d) AC charge current $\left|I_{2}^{\Delta_{2}=0}\right|$ in the absence of the Higgs mode, i.e., by artificially setting $\Delta_{2}=0$. Panels (e) and (f) are line cuts of (d) at different $\Omega$ and $\mathrm{eV}$, respectively. The charge currents are in units of $G_{t} W_{A} / e$. The temperature is $T=$ $0.05 T_{c}$; the other parameters are the same as in Fig. 1(b).

up to around $e V=\Delta_{0}$, then starts to decrease. At resonance $\Omega=\Delta_{0}$, the AC charge current shows a peak around $e V=$ $\Delta_{0}$. This is explained as follows. The Higgs mode can be interpreted as coherent depairing and pairing of Cooper pairs. The characteristic frequency of the Higgs mode is $2 \Delta_{0}$, thus the split quasiparticles (or depaired Cooper pairs) will appear around the SC band edge. Since it is the split quasiparticles that contribute to the $\mathrm{AC}$ charge current at resonance, the $\mathrm{AC}$ charge current reaches a maximum if the DC voltage bias aligns with the SC band edge at low temperatures. There is also a kink at $\mathrm{eV}=3 \Delta_{0}$ that can be interpreted as a side band due to the Higgs mode that modulates the quasiparticle density.

Away from resonance, we can observe some other special points in Fig. 2(b). These points are due to the excitation from the electromagnetic irradiation with maxima at $e V=$ $\Delta_{0},-\Delta_{0}+2 \Omega, \Delta_{0}+\Omega$ and a kink at $\Delta_{0}+2 \Omega$ as can be observed from Fig. 2(e). At higher temperatures, the nonmonotonic behavior of $\left|I_{2}\right|$ still survives, while the maximum point shifts to higher voltages with $e V>\Delta_{0}$ due to thermal 
excitations (see Fig. S2 in the Supplemental Material [41] for $T=0.7 T_{c}$ )

Under a large bias, the AC charge current $I_{2}$ shows characteristic features: even at $e V=6 \Delta_{0}$, a resonant behavior as a function of $\Omega$ can be observed in Fig. 2(c). In contrast, if the Higgs mode is not taken into account, the AC charge current $I_{2}^{\Delta_{2}=0}$ vanishes at large voltage bias even for $\Omega=\Delta_{0}$ as shown in Fig. 2(e). At resonance and with $e V \gg \Delta_{0}$, an analytical estimate given in the Supplemental Material [41] shows that the $\mathrm{AC}$ current is inversely proportional to the voltage bias, i.e., $I_{2} \propto \Delta_{2} /(\mathrm{eV})$. Thus a finite $\mathrm{AC}$ charge current can persist up to relatively large voltage biases. This characteristic behavior of the $\mathrm{AC}$ charge current at large bias near resonance can serve as an indicator for the presence of the Higgs mode in the superconductor.

Apart from the possibility to tune the irradiation frequency continuously in experiment $[45,46]$, one can alternatively tune the Higgs mode to be at or away from the resonant condition by changing the system temperature [22]. In Fig. 1(c), we show the AC charge current magnitude as a function of voltage bias and temperature. The electromagnetic frequency is fixed at $\Omega=\left.\left.\Delta_{0}\right|_{T=0.7 T_{c}} \approx 0.8 \Delta_{0}\right|_{T=0}$, which means that the resonance is achieved by tuning the temperature to be at $T=0.7 T_{c}$. This leads to a prominent resonant behavior as shown in Fig. 1(c).

Figure 1(d) also shows that $\left|I_{2}\right|$ exhibits a nonmonotonic behavior as a function of voltage bias. At a low temperature with $T=0.01 T_{c},\left|I_{2}\right|$ reaches its maximum around $e V=\Delta_{0}$. It also has maxima at both $0.6 \Delta_{0}\left(\approx-\Delta_{0}+2 \Omega\right)$ and $1.8 \Delta_{0}$ $\left(\approx \Delta_{0}+\Omega\right)$ and a kink at $2.6 \Delta_{0}\left(\approx \Delta_{0}+2 \Omega\right)$ due to photonassisted transport processes. Due to thermal excitations, the voltage bias at which $I_{2}$ reaches its maximum increases with increasing temperature. Once the temperature reaches the SC critical temperature, both the static SC gap amplitude and its oscillation vanish, and so does the AC charge current. Similarly to Fig. 2(b), we observe a slow decay $\propto \Delta_{2} /(\mathrm{eV})$ of the AC charge current at large bias near resonance.

Since $\Delta_{2}$ is proportional to $W_{A}$ [41], the next-order correction to the time-dependent order parameter $\Delta(t)$ in Eq. (1) is proportional to $W_{A}^{2}$. Higher-order corrections to $\Delta(t)$ can be ignored if $W_{A} / \Delta_{0} \ll 1$ is satisfied. Given the smallness of $W_{A} /\left(\left.\Delta_{0}\right|_{T=0}\right)$, which is 0.01 in this work, the condition $W_{A} / \Delta_{0} \ll 1$ can be satisfied if the temperature is not too close to $T_{c}$.

The AC current $I_{2}$ is given in units of $G_{t} W_{A} / e$ where $G_{t}$ is the tunnel conductance of the junction and the value chosen for the coupling parameter $W_{A}$ is $W_{A}=\left.0.01 \Delta_{0}\right|_{T=0}$. For example, for the superconductor $\mathrm{NbN},\left.\Delta_{0}\right|_{T=0} \approx 2.6 \mathrm{meV}$ [31], so that the values of $I_{2}$ shown in the figures are approximately in units of $0.026 \mathrm{mV} \mathrm{G}_{t}$.

Discussion. The physical picture that emerges is as follows: the pairing/depairing processes associated with the orderparameter oscillation (Higgs mode) will create particles and holes each of which contribute to the AC tunnel current. At zero voltage bias, their respective contributions cancel. A finite voltage bias leads to particle-hole asymmetry that results in a finite $\mathrm{AC}$ current that exhibits signatures of the Higgs mode. On the one hand, this AC charge current may be measured directly. Alternatively, the NS junction can be experimentally manufactured as an antenna, where the electromagnetic irradiation magnitude and frequency due to the AC charge current can be detected. Even though a tunnel junction is studied here, we expect all of the discussed features to be present for a highly transparent junction as well. Note that it is well established that nonequilibrium achieved by voltage biasing can affect the AC response of a superconductor (see, e.g., [47]). However, our work considers the inverse situation that the AC response of a superconductor (the Higgs mode) can affect the transport properties of a voltage-biased junction.

The order-parameter oscillation will slowly decay in a power-law way if the electromagnetic irradiation is switched off, and the same applies to the AC charge current. Here, we only treat the steady-state situation with a constant electromagnetic irradiation.

At low temperatures $T \ll \Delta_{0}$, the conductance of the NS junction that we consider is exponentially suppressed. Thus, for the results presented in Fig. 2, where the temperature is chosen as $T=0.05 T_{C}$, we do not expect Joule heating to be a severe problem, and the predicted effects will be unchanged. For the results presented in Figs. 1(c) and 1(d), if Joule heating exists, the resonant behavior will occur at a lower temperature. However, we expect all the qualitative features to remain.

In the work presented above, the wave vector of the electromagnetic field is assumed to be parallel to the transport direction of the junction. If the wave vector has a finite orthogonal component, the supercurrent, which can be induced by the Andreev reflection processes, will mediate a linear coupling between the Higgs mode and the electromagnetic field [30-33]. In this case, the time-dependent order parameter can be written as $\Delta(t)=\Delta_{0}+\Delta_{1} e^{-i \Omega t}+\Delta_{2} e^{-i 2 \Omega t}$ with an additional term of $\Delta_{1}$ compared to Eq. (1). $\Delta_{1}$ and $\Delta_{2}$ exhibit resonances at $\Omega=2 \Delta_{0}$ and $\Omega=\Delta_{0}$, respectively. AC charge transport in an NS junction in the presence of a linear coupling between the Higgs mode and the electromagnetic irradiation will be investigated in the future. The generalization to an NS junction with unconventional superconductors $[27,37,38]$ may also be interesting.

Conclusion. We have studied the AC transport properties of a DC-voltage-biased NS tunnel junction taking into account the Higgs mode in the superconductor. The pronounced resonant behavior, the characteristic nonmonotonic behavior of the AC charge current with increasing bias, and its slow decay inversely proportional to the bias can serve as signatures of the presence of the Higgs mode.

Our results could be applied to design more complex superconducting devices featuring the Higgs dynamics in coupled junctions of superconducting islands. Furthermore, it will be interesting to combine Josephson effects with the Higgs mode.

Acknowledgments. G.T. and C.B. acknowledge financial support from the Swiss National Science Foundation (SNSF) and the NCCR Quantum Science and Technology. 
[1] A. F. G. Wyatt, V. M. Dmitriev, W. S. Moore, and F. W. Sheard, Microwave-Enhanced Critical Supercurrents in Constricted Tin Films, Phys. Rev. Lett. 16, 1166 (1966).

[2] A. H. Dayem and J. J. Wiegand, Behavior of thin-film superconducting bridges in a microwave field, Phys. Rev. 155, 419 (1967).

[3] J.-J. Chang and D. J. Scalapino, Gap enhancement in superconducting thin films due to microwave irradiation, J. Low Temp. Phys. 29, 477 (1977).

[4] K. S. Tikhonov, M. A. Skvortsov, and T. M. Klapwijk, Superconductivity in the presence of microwaves: Full phase diagram, Phys. Rev. B 97, 184516 (2018).

[5] J. B. Curtis, Z. M. Raines, A. A. Allocca, M. Hafezi, and V. M. Galitski, Cavity Quantum Eliashberg Enhancement of Superconductivity, Phys. Rev. Lett. 122, 167002 (2019).

[6] H. Dehghani, Z. M. Raines, V. M. Galitski, and M. Hafezi, Optical enhancement of superconductivity via targeted destruction of charge density waves, Phys. Rev. B 101, 224506 (2020).

[7] D. Fausti, R. I. Tobey, N. Dean, S. Kaiser, A. Dienst, M. C. Hoffmann, S. Pyon, T. Takayama, H. Takagi, and A. Cavalleri, Light-induced superconductivity in a stripe-ordered cuprate, Science 331, 189 (2011).

[8] M. Mitrano, A. Cantaluppi, D. Nicoletti, S. Kaiser, A. Perucchi, S. Lupi, P. Di Pietro, D. Pontiroli, M. Riccò, S. R. Clark, D. Jaksch, and A. Cavalleri, Possible light-induced superconductivity in $\mathrm{K}_{3} \mathrm{C}_{60}$ at high temperature, Nature (London) 530, 461 (2016).

[9] F. Schlawin, A. Cavalleri, and D. Jaksch, Cavity-Mediated Electron-Photon Superconductivity, Phys. Rev. Lett. 122, 133602 (2019).

[10] O. Hart, G. Goldstein, C. Chamon, and C. Castelnovo, Steadystate superconductivity in electronic materials with repulsive interactions, Phys. Rev. B 100, 060508(R) (2019).

[11] M. Claassen, D. M. Kennes, M. Zingl, M. A. Sentef, and A. Rubio, Universal optical control of chiral superconductors and Majorana modes, Nat. Phys. 15, 766 (2019).

[12] P. W. Anderson, Plasmons, gauge invariance, and mass, Phys. Rev. 130, 439 (1963).

[13] C. M. Varma, Higgs boson in superconductors, J. Low Temp. Phys. 126, 901 (2002).

[14] D. Pekker and C. Varma, Amplitude/Higgs modes in condensed matter physics, Ann. Rev. Condens. Matter Phys. 6, 269 (2015).

[15] P. W. Anderson, Higgs, Anderson and all that, Nat. Phys. 11, 93 (2015).

[16] R. Sooryakumar and M. V. Klein, Raman Scattering by Superconducting-Gap Excitations and Their Coupling to Charge-Density Waves, Phys. Rev. Lett. 45, 660 (1980).

[17] R. Sooryakumar and M. V. Klein, Raman scattering from superconducting gap excitations in the presence of a magnetic field, Phys. Rev. B 23, 3213 (1981).

[18] R. Grasset, T. Cea, Y. Gallais, M. Cazayous, A. Sacuto, L. Cario, L. Benfatto, and M.-A. Méasson, Higgs-mode radiance and charge-density-wave order in $2 \mathrm{H}$ - $\mathrm{NbSe}_{2}$, Phys. Rev. B 97, 094502 (2018).

[19] P. B. Littlewood and C. M. Varma, Gauge-Invariant Theory of the Dynamical Interaction of Charge Density Waves and Superconductivity, Phys. Rev. Lett. 47, 811 (1981).

[20] P. B. Littlewood and C. M. Varma, Amplitude collective modes in superconductors and their coupling to charge-density waves, Phys. Rev. B 26, 4883 (1982).
[21] R. Matsunaga, Y. I. Hamada, K. Makise, Y. Uzawa, H. Terai, Z. Wang, and R. Shimano, Higgs Amplitude Mode in the BCS Superconductors $\mathrm{Nb}_{1-x} \mathrm{Ti}_{x} \mathrm{~N}$ Induced by Terahertz Pulse Excitation, Phys. Rev. Lett. 111, 057002 (2013).

[22] R. Matsunaga, N. Tsuji, H. Fujita, A. Sugioka, K. Makise, Y. Uzawa, H. Terai, Z. Wang, H. Aoki, and R. Shimano, Lightinduced collective pseudospin precession resonating with Higgs mode in a superconductor, Science 345, 1145 (2014).

[23] A. F. Kemper, M. A. Sentef, B. Moritz, J. K. Freericks, and T. P. Devereaux, Direct observation of Higgs mode oscillations in the pump-probe photoemission spectra of electron-phonon mediated superconductors, Phys. Rev. B 92, 224517 (2015).

[24] N. Tsuji and H. Aoki, Theory of Anderson pseudospin resonance with Higgs mode in superconductors, Phys. Rev. B 92, 064508 (2015).

[25] H. P. Ojeda Collado, J. Lorenzana, G. Usaj, and C. A. Balseiro, Population inversion and dynamical phase transitions in a driven superconductor, Phys. Rev. B 98, 214519 (2018).

[26] R. Shimano and N. Tsuji, Higgs mode in superconductors, Ann. Rev. Condens. Matter Phys. 11, 103 (2020).

[27] K. Katsumi, N. Tsuji, Y. I. Hamada, R. Matsunaga, J. Schneeloch, R. D. Zhong, G. D. Gu, H. Aoki, Y. Gallais, and R. Shimano, Higgs Mode in the $d$-wave Superconductor $\mathrm{Bi}_{2} \mathrm{Sr}_{2} \mathrm{CaCu}_{2} \mathrm{O}_{8+x}$ Driven by an Intense Terahertz Pulse, Phys. Rev. Lett. 120, 117001 (2018).

[28] M. A. Silaev, R. Ojajärvi, and T. T. Heikkilä, Spin currents driven by the Higgs mode in magnetic superconductors, arXiv:1907.00539.

[29] H. Krull, N. Bittner, G. S. Uhrig, D. Manske, and A. P. Schnyder, Coupling of Higgs and Leggett modes in non-equilibrium superconductors, Nat. Commun. 7, 11921 (2016).

[30] A. Moor, A. F. Volkov, and K. B. Efetov, Amplitude Higgs Mode and Admittance in Superconductors with a Moving Condensate, Phys. Rev. Lett. 118, 047001 (2017).

[31] S. Nakamura, Y. Iida, Y. Murotani, R. Matsunaga, H. Terai, and R. Shimano, Infrared Activation of the Higgs Mode by Supercurrent Injection in Superconducting NbN, Phys. Rev. Lett. 122, 257001 (2019).

[32] Z. M. Raines, A. A. Allocca, M. Hafezi, and V. M. Galitski, Cavity Higgs polaritons, Phys. Rev. Research 2, 013143 (2020).

[33] M. Puviani, L. Schwarz, X.-X. Zhang, S. Kaiser, and D. Manske, Current-assisted Raman activation of the Higgs mode in superconductors, Phys. Rev. B 101, 220507(R) (2020).

[34] V. L. Vadimov, I. M. Khaymovich, and A. S. Mel'nikov, Higgs modes in proximized superconducting systems, Phys. Rev. B 100, 104515 (2019).

[35] H. Uematsu, T. Mizushima, A. Tsuruta, S. Fujimoto, and J. A. Sauls, Chiral Higgs Mode in Nematic Superconductors, Phys. Rev. Lett. 123, 237001 (2019).

[36] M. Buzzi, G. Jotzu, A. Cavalleri, J. I. Cirac, E. A. Demler, B. I. Halperin, M. D. Lukin, T. Shi, Y. Wang, and D. Podolsky, Higgs-mediated optical amplification in a non-equilibrium superconductor, arXiv:1908.10879.

[37] L. Schwarz, B. Fauseweh, N. Tsuji, N. Cheng, N. Bittner, H. Krull, M. Berciu, G. S. Uhrig, A. P. Schnyder, S. Kaiser, and D. Manske, Classification and characterization of nonequilibrium Higgs modes in unconventional superconductors, Nat. Commun. 11, 287 (2020). 
[38] F. Yang and M. W. Wu, Theory of Higgs modes in $d$-wave superconductors, arXiv:2001.06183.

[39] W. Belzig, F. K. Wilhelm, C. Bruder, G. Schön, and A. D. Zaikin, Quasiclassical Green's function approach to mesoscopic superconductivity, Superlattices Microstruct. 25, 1251 (1999).

[40] N. Kopnin, Theory of Nonequilibrium Superconductivity (Oxford University Press, New York, 2001).

[41] See Supplemental Material at http://link.aps.org/supplemental/ 10.1103/PhysRevResearch.2.022068 for derivations and additional details.

[42] Y. V. Nazarov, Novel circuit theory of Andreev reflection, Superlattices Microstruct. 25, 1221 (1999).

[43] J. Börlin, W. Belzig, and C. Bruder, Full Counting Statistics of a Superconducting Beam Splitter, Phys. Rev. Lett. 88, 197001 (2002).
[44] F. S. Bergeret, P. Virtanen, A. Ozaeta, T. T. Heikkilä, and J. C. Cuevas, Supercurrent and Andreev bound state dynamics in superconducting quantum point contacts under microwave irradiation, Phys. Rev. B 84, 054504 (2011).

[45] B. Liu, H. Bromberger, A. Cartella, T. Gebert, M. Först, and A. Cavalleri, Generation of narrowband, high-intensity, carrierenvelope phase-stable pulses tunable between 4 and $18 \mathrm{THz}$, Opt. Lett. 42, 129 (2017).

[46] B. Liu, M. Först, M. Fechner, D. Nicoletti, J. Porras, T. Loew, B. Keimer, and A. Cavalleri, Pump Frequency Resonances for Light-Induced Incipient Superconductivity in $\mathrm{YBa}_{2} \mathrm{Cu}_{3} \mathrm{O}_{6.5}$, Phys. Rev. X 10, 011053 (2020).

[47] G. Catelani, L. I. Glazman, and K. E. Nagaev, Effect of quasiparticles injection on the ac response of a superconductor, Phys. Rev. B 82, 134502 (2010). 